\title{
Relações interespecíficas entre parasitoides nativos de moscas-das-frutas e o braconídeo exótico Diachasmimorpha longicaudata em frutos de 'umbu-cajá'
}

\author{
Interespecific relations between native parasitoids of fruit flies and exotic braconid Diachasmimorpha \\ longicaudata in fruits of 'umbu-cajá'
}

\author{
Zuzinaide Vidal Bomfim $^{\mathrm{I}^{*}}$ Romulo da Silva Carvalho"I Carlos Alfredo Lopes de Carvalho $^{\mathrm{I}}$
}

RESUMO

Espécies de vespas parasitoides (Hymenoptera: Braconidae) são importantes agentes de controle biológico de moscas-das-frutas (Diptera: Tephritidae). Este trabalho teve por objetivo conhecer os efeitos da liberação e as relações de competitividade interespecífica do parasitoide exótico Diachasmimorpha longicaudata Ashmead sobre o complexo de parasitoides nativos de moscas-das-frutas associado a frutos de 'umbu-cajá' (Spondias spp.) na região do Recôncavo Baiano. Entre os meses de abril e julho de 2006, 8.955 frutos $(192,93 \mathrm{~kg})$ foram coletados antes e após (24 e 48 horas) a liberação de 9.600 fêmeas de D. longicaudata em campo. Obteve-se um total de 8.724 pupários de Tephritidae, dos quais emergiram 3.963 adultos de Anastrepha obliqua (Macquart) e 1.115 parasitoides. A maior frequência relativa foi de Doryctobracon areolatus (Szépligeti), seguida por Asobara anastrephae (Muesebeck) e Utetes anastrephae (Viereck). Após 24 e 48 horas da liberação do parasitoide exótico D. longicaudata em campo, constatou-se que o índice de parasitismo total aumentou de 15,86 para 20,4 e $45,19 \%$, respectivamente. Assim, observou-se que a liberação da espécie exótica D. longicaudata não apresenta efeitos negativos na ocorrência dos parasitoides nativos e contribui para complementar o controle biológico natural de A. obliqua em frutos de 'umbu-cajá', nas condições deste estudo.

Palavras-chave: Anastrepha spp., Braconidae, controle biológico, liberação em campo, Spondias spp.

\section{ABSTRACT}

Wasps parasitoid species (Hymenoptera: Braconidae) are fruit flies (Diptera: Tephritidae) biological control important agents. This study aimed to know the effects of the release and interspecific competitive relationships of the exotic parasitoid Diachasmimorpha longicaudata Ashmead (Hymenoptera: Braconidae) on the native parasitoid complex of fruit flies in Spondias spp. in the region of Recôncavo Baiano. From April to July of 2006, 8.955 fruits (192.93kg) were collected before and after (24 and 48 hours) release of 9.600 females of D. Iongicaudata. Exactly 8.724 Tephritidae pupae were obtained, of which 3.963 Anastrepha obliqua (Macquart) adults and 1.115 parasitoids of Braconidae family emerged. Three species of opiinae were identified before releasing. The higher relative frequency was of Doryctobracon areolatus (Szépligeti), followed by Asobara anastrephae (Muesebeck) and Utetes anastrephae (Viereck). 24 and 48 hours after release the exotic parasitoid $\mathbf{D}$. longicaudata on the field, the parasitism rate increased from $15.86 \%$ to $20.42 \%$ and $45.19 \%$, respectively. It was found that the release of exotic species $\boldsymbol{D}$. longicaudata has no negative effects on the occurrence of native parasitoids and helps to complement the natural biological control of A. obliqua in fruits of 'umbu-cajá' conditions of this study.

Key words: Anastrepha spp., Braconidae, Biological control, release in field, Spondias spp.

\section{INTRODUÇÃO}

O parasitoide exótico Diachasmimorpha longicaudata (Ashmead) (Hymenoptera: Braconidae), sinonímia (Opius longicaudatus Ashmead e Biosteres longicaudatus Ashmead), é um endoparasitoide originário da região indo-australiana e está entre as cinco espécies de braconídeos da subfamília Opiinae, de importância na regulação das populações de moscasdas-frutas, parasitando preferencialmente larvas de $2^{\circ}$ e 3o estádio (PURCELL et al., 1994). Esse parasitoide tem sido a espécie mais utilizada, em nível mundial, no

ICentro de Ciências Agrárias, Ambientais e Biológicas, Universidade Federal do Recôncavo da Bahia (UFRB), 44380-000, Cruz das

Almas, BA, Brasil. E-mail: zuzividal@yahoo.com.br. *Autor para correspondência.

IEMBRAPA Mandioca e Fruticultura Tropical, Cruz das Almas, BA, Brasil. 
controle biológico clássico de moscas frugívoras, com exemplos de utilização prática em diversos países. $\mathrm{Na}$ Flórida, por exemplo, essa espécie destaca-se pela facilidade de criação em laboratório e rápida adaptação aos ambientes onde é liberada, bem como pela condição de especialista no parasitismo de grande número de espécies de tefritídeos (BARANOWSKI et al., 1993).

Em setembro de 1994, o Brasil importou o parasitoide exótico $\boldsymbol{D}$. Iongicaudata, oriundo do Departament of Plant Industry, Gainesville, Flórida (EUA), pela EMBRAPA Mandioca e Fruticultura Tropical, com apoio do Laboratório de Quarentena Costa Lima da Embrapa Meio Ambiente. O objetivo da introdução foi avaliar o potencial de utilização desse agente de controle biológico no manejo das moscasdas-frutas neotropicais do gênero Anastrepha e da espécie exótica Ceratitis capitata (CARVALHO \& NASCIMENTO, 2002).

A introdução de uma espécie exótica, além de levantar dúvidas em relação a sua eficiência, pode provocar competição interespecífica com deslocamento de nichos, perda da biodiversidade e ocorrência de hiperparasitismo (BENNET, 1993; DUAN et al., 1997). Tradicionalmente, a medida do impacto causada pelos parasitoides introduzidos é feita com base no índice de parasitismo que permite avaliar a mortalidade que o inimigo natural pode causar sobre a população de determinada espécie hospedeira (WAAGE \& MILLS, 1992). Assim, é fundamental a realização de levantamentos prévios das espécies nativas antes de qualquer introdução de um inimigo natural exótico, pois a falta de informações sobre a bioecologia de inimigos naturais em seu habitat pode levar a falhas no estabelecimento de uma espécie introduzida e/ou trazer os efeitos negativos salientados anteriormente.

Em estudos que envolvem relações tritróficas com moscas-das-frutas, a planta hospedeira assume grande importância, pois influenciará a atuação do inseto relacionado ao terceiro nível trófico (e.g. parasitoides). Na região do Recôncavo Baiano, a 'umbucajá' (Spondias spp.) destaca-se como excelente modelo ao estudo, pois, além de ser hospedeira de espécies da Família Tephritidae, levantamentos prévios indicam a existência de um complexo de parasitoides nativos associados, o que facilita a compreensão das relações de competitividade (CARVALHO, 2005).

Dentro desse contexto, este trabalho teve como objetivo conhecer o efeito da liberação e as relações de competitividade interespecífica do parasitoide exótico $\boldsymbol{D}$. longicaudata sobre o complexo de parasitoides nativos de moscas-das-frutas associados a frutos de 'umbu-cajá' na região do Recôncavo Baiano.

\section{MATERIAL E MÉTODOS}

Característica da região - O experimento foi realizado durante o período de abril a julho de 2006, na região do Recôncavo Baiano, nos Municípios de Cabaceiras do Paraguaçu e Cruz das Almas, Bahia (BA). $\mathrm{O}$ clima da região é tropical quente úmido. Durante o período de realização do estudo, a precipitação média do ano foi de $41,1 \mathrm{~mm}$, a temperatura média foi de $27,1^{\circ} \mathrm{C}$, e umidade relativa foi de 80\% (AGRITEMPO, 2009).

Criação do parasitoide $\boldsymbol{D}$. longicaudata em laboratório e liberação de fêmeas

A partir de uma colônia pré-estabelecida no Laboratório de Entomologia da Embrapa Mandioca e Fruticultura Tropical, o parasitoide D. longicaudata foi multiplicado, com base no método de criação descrito por CARVALHO \& NASCIMENTO (2002), em condições controladas de temperatura, $25 \pm 1^{\circ} \mathrm{C}$; umidade relativa: $60 \pm 10 \%$ e fotofase de 14 horas.

Ao atingirem nove dias de idade, as fêmeas do parasitoide exótico foram aspiradas da colônia de criação em laboratório, acondicionadas em potes plásticos e transportadas em caixa de isopor para o campo. Os potes foram colocados sobre os galhos de nove árvores de 'umbu-cajá', em fase de frutificação, previamente selecionadas nos Municípios de Cruz das Almas e Cabaceiras do Paraguaçu. Foi estabelecido o horário da manhã para a realização das liberações, sendo os potes mantidos sob constante observação visual até a total ausência de fêmeas no interior destes. O número total de fêmeas do parasitoide exótico liberadas variou, em cada local, de acordo com a sua disponibilidade no laboratório (Tabela 1).

Levantamento prévio, monitoramento e obtenção de adultos

Foram amostrados frutos maduros de umbucajá mediante a coleta sob a copa das fruteiras. Os frutos foram coletados antes da liberação e após 24 e 48 horas da liberação inoculativa de fêmeas do parasitoide exótico em campo. O número de frutos coletados variou de acordo com a disponibilidade nas plantas. Os frutos coletados foram transportados para o laboratório, onde foram quantificados, pesados, acondicionados em bandejas contendo vermiculita (substrato) e mantidos em observação até a obtenção de larvas e/ou pupários. As moscas e/ou parasitoides emergidos de cada amostra foram preservados em álcool 70\% para posterior identificação taxonômica. 
Relações interespecíficas entre parasitoides nativos de moscas-das-frutas e o braconídeo exótico...

Tabela 1 - Localização das fruteiras de '’umbu-cajá' (Spondias spp.) e densidade de fêmeas do parasitoide exótico D. Iongicaudata, liberadas em dois municípios do Recôncavo Baiano. Abril a Julho de 2006.

\begin{tabular}{|c|c|c|c|c|}
\hline Local & Número de plantas & $\begin{array}{l}\text { Densidade de liberação } \\
\text { (Adultos/planta) }\end{array}$ & $\begin{array}{l}\text { Total de fêmeas de } \boldsymbol{D} \text {. } \\
\text { longicaudata }\end{array}$ & Localização geográfica \\
\hline \multirow{3}{*}{ Cabaceiras do Paraguaçu } & 4 & 1.150 & 4.600 & $\mathrm{~S} 12^{\circ} 33^{\prime}$ \\
\hline & & & & $\mathrm{W} 39^{\circ} 08^{\prime}$ \\
\hline & 5 & 960 & 4.800 & $\mathrm{~S} 12^{\circ} 39^{\prime}$ \\
\hline \multirow[t]{2}{*}{ Cruz das Almas } & & & & W $39^{\circ} 05^{\prime}$ \\
\hline & & & & ---- \\
\hline Total & 9 & - & 9.600 & - \\
\hline
\end{tabular}

Identificação taxonômica

As fêmeas de moscas-das-frutas foram identificadas com base na metodologia descrita por ZUCCHI (2000), e os parasitoides foram identificados de acordo com SOUZA FILHO (1999). As espécies de parasitoides e de moscas-das-frutas foram depositadas no Museu Entomológico do Centro de Ciências Agrárias, Ambientais e Biológicas da Universidade Federal do Recôncavo da Bahia (UFRB), em Cruz das Almas, BA.

Cálculo do índice - Para analisar as relações entre as espécies estudadas, foram adotados o índice de parasitismo total (PT) e a frequência relativa por espécies de parasitoides (E), conforme MATRANGOLO et al. (1998), por meio das seguintes equações: PT\% = ( $\mathrm{n}^{\circ}$ de parasitoides emergidos) / ( $\mathrm{n}^{\circ}$ de moscas emergidas $+n^{\circ}$ parasitoides emergidos) $X 100$; $\mathrm{E} \%=$ ( $\mathrm{n}^{\circ}$ indivíduos de uma espécie) / $\mathrm{n}$ - total de parasitoides emergidos X 100. Os cálculos foram efetuados antes e após (24 e 48 horas) da liberação das fêmeas do parasitoide exótico $\boldsymbol{D}$. longicaudata, sob a copa das fruteiras de umbu-cajá. Os resultados foram submetidos à análise de variância, utilizando-se o software Sisvar v.4.6 (FERREIRA, 2004). As médias dos índices de parasitismo obtidos antes e após (24 e 48 horas) da liberação de $\boldsymbol{D}$. longicaudata foram comparadas entre si pelo Teste de Tukey, a 5\% de probabilidade.

\section{RESULTADOS E DISCUSSÃO}

Do total de 8.955 (192,93kg) frutos de 'umbucajá' coletados durante as três etapas de estudo, foram obtidos 8.724 pupários, os quais apresentaram uma viabilidade pupal total de 45,43\%. Desse total, emergiram dípteros frugívoros da família Tephritidae, sendo 3.963 indivíduos de Anastrepha obliqua e dois exemplares de Neosilba spp. (Lonchaeidae). Constatou-se que todos os 1.115 parasitoides emergidos, obtidos a partir do somatório das diferentes fases do experimento, pertenciam à família Braconidae (Tabela 2).

Durante a realização da primeira etapa do estudo, quando foi realizado o levantamento prévio do complexo de parasitoides nativos antes da liberação de fêmeas de $\boldsymbol{D}$. longicaudata em campo, foram obtidos 64,82\% de braconídeos representantes da subfamília Opiinae (Doryctobracon areolatus (Szépligeti) (63,81\%), Utetes anastrephae (Viereck) (1,01\%) e 35,18\% de Asobara anastrephae (Muesebeck) (Alysiinae).

Antes da liberação de $\boldsymbol{D}$. longicaudata em campo, apenas três espécies de parasitoides foram registradas, A. anastrephae, $D$. areolatus e $U$. anastrephae, no Município de Cruz das Almas, sendo apenas as duas primeiras registradas no Município de Cabaçeiras do Paraguaçu. D. areolatus e $\boldsymbol{A}$. anastrephae foram encontradas com 22 e $78 \%$ de frequência no Município de Cabaceiras do Paraguaçu,

Tabela 2 - Número total de frutos, pupários, moscas emergidas e parasitoides coletados nas três etapas de estudo (antes e após 24 e 48 horas da liberação do parasitoide D. longicaudata), nos Municípios de Cabaceiras do Paraguaçu e Cruz das Almas, na região do Recôncavo Baiano. Abril a julho 2006.

\begin{tabular}{lccccc}
\hline Municípios do Recôncavo Baiano & № de frutos & № de pupários & \multicolumn{2}{c}{ № de moscas emergidas } & No de parasitoides (Braconidae) \\
& & & A. obliqua & Neosilba sp. \\
\hline Cabaçeiras do Paraguaçu & 3.335 & 2.499 & 1.045 & 00 & 02 \\
Cruz das Almas & 5.620 & 6.225 & 2.912 & 02 & 551 \\
Total & 8.955 & 8.724 & 3.957 & 1.115 \\
\hline
\end{tabular}


diferentemente dos resultados obtidos no Município de Cruz das Almas, onde foram registrados 39,6 e 59,0\%, respectivamente. Já $\boldsymbol{U}$. anastrephae foi a menor frequência observada em Cabaceiras do Paraguaçu, com 1,3\% (Tabela 3).

A ocorrência de $\boldsymbol{D}$. areolatus neste trabalho corrobora a frequência dessa espécie parasitando larvas de tefritídeos em frutos de umbu-cajá coletados em vários municípios da região do Recôncavo Baiano, a qual foi encontrada com 94,10\% de frequência quando comparada às demais espécies de parasitoides nativos (SANTOS et al., 2008). Esse fato provavelmente está relacionado com a antecipação dessa espécie em localizar as larvas no interior de diferentes frutos hospedeiros (MATRANGOLO et al., 1998) e apresentar ovipositor mais longo que A. anastrephae e $\boldsymbol{U}$. anastrephae (SIVINSK et al., 1997).

A espécie nativa, $A$. anastrephae, foi a segunda mais coletada antes da liberação e 24 horas após, com 14,0\% no Município de Cabaceiras do Paraguaçu e 14,1\% em Cruz das Almas (Tabela 3). Ao analisar a frequência relativa desse alysiíneo, 48 horas após a liberação, foi possível constatar que essa espécie continuou sendo a segunda em frequência, com 27,1\% em Cabaceiras do Paraguaçu e 30,2\% em Cruz das Almas (Tabela 3).

Em Cabaceiras do Paraguaçu, além de $\boldsymbol{D}$. areolatus e A. anastrephae, foi detectada a presença de $\boldsymbol{U}$. anastrephae (1,17\%) e, em Cruz das Almas, a ocorrência do braconídeo nativo, Opius sp. (0,7\%). A espécie exótica foi recapturada, sendo a terceira em frequência, 5,8\% em Cabaceiras do Paraguaçu e 4,4\% em Cruz das Almas (Tabela 3).

A frequência de $\boldsymbol{D}$. longicaudata no estudo, após 48 horas da sua liberação, foi relativamente baixa quando comparada com os resultados obtidos após 24 horas. No entanto, a espécie nativa $\boldsymbol{D}$. areolatus continuou sendo a mais frequente, com relativo aumento em 24 horas. Essa espécie apresentou alterações na sua frequência relativa após 48 horas da liberação (Tabela 3).

Esse resultado provavelmente ocorreu em razão das relações de competição interespecífica pelo mesmo sítio de oviposição. Em estudo realizado no Município de Conceição do Almeida, BA, constatouse, após 17 meses de monitoramento, que a competição imposta pelo complexo de parasitoides nativos ao braconídeo exótico, aliada ao pequeno número de $\boldsymbol{D}$. longicaudata liberado de forma inoculativa, não permitiram o seu estabelecimento efetivo na área liberada. Apesar disso, a espécie exótica foi capaz de se manter no agroecossistema, sendo recuperados indivíduos um ano e cinco meses após o término de sua liberação, o que reforça a sua capacidade de parasitar espécies de moscas-das-frutas neotropicais, sobrevivendo e mantendo-se por várias gerações nas condições desse ambiente tropical (CARVALHO, 2005).

Por outro lado, quando liberado em um pomar de goiaba no município de Minas Gerais, verificou-se que $\boldsymbol{D}$. longicaudata não ocasionou redução no número do parasitoide nativo e sim um aumento, evidenciando que não houve competição pelo nicho (hospedeiro) (ALVARENGA et al., 2005). No entanto, neste estudo, apesar do pequeno número liberado, a espécie exótica foi a terceira encontrada parasitando larvas de tefritídeos em frutos de umbucajá, nos dois municípios do Recôncavo Baiano (Tabela 3), fato que evidencia a relação de competição pelo mesmo nicho (hospedeiro), como citado anteriormente. Apesar de a espécie exótica $\boldsymbol{D}$. longicaudata apresentar algumas características semelhantes à espécie nativa $\boldsymbol{D}$. areolatus, como, por

Tabela 3 - Frequência (\%) e número de espécies de parasitoides antes e após 24 e 48 horas da liberação de fêmeas do parasitoide exótico $\boldsymbol{D}$. longicaudata nos municípios de Cabaceiras do Paraguaçu e Cruz das Almas, na região do Recôncavo Baiano. Abril a julho 2006.

\begin{tabular}{|c|c|c|c|c|c|c|c|c|c|c|c|c|c|c|c|}
\hline \multirow{2}{*}{$\begin{array}{l}\text { Municípios do } \\
\text { Recôncavo Baiano }\end{array}$} & \multicolumn{5}{|c|}{ Antes da liberação $(\mathrm{T}=0)^{1}$} & \multicolumn{5}{|c|}{24 horas após liberação } & \multicolumn{5}{|c|}{48 horas após liberação } \\
\hline & $\mathrm{Da}$ & Aa & $\mathrm{Ua}$ & Osp & $\mathrm{Dl}$ & $\mathrm{Da}$ & Aa & $\mathrm{Ua}$ & Osp & $\mathrm{Dl}$ & $\mathrm{Da}$ & $\mathrm{Aa}$ & $\mathrm{Ua}$ & Osp & $\mathrm{Dl}$ \\
\hline $\begin{array}{l}\text { Cabaçeiras do } \\
\text { Paraguaçu }\end{array}$ & $\begin{array}{l}78,0 \\
(39)\end{array}$ & $\begin{array}{l}22,0 \\
(11)\end{array}$ & - & - & - & $\begin{array}{c}78,9 \\
(135)\end{array}$ & $\begin{array}{l}14,0 \\
(24)\end{array}$ & $\begin{array}{l}1,17 \\
(02)\end{array}$ & - & $\begin{array}{c}5,8 \\
(10)\end{array}$ & $\begin{array}{c}69,0 \\
(237)\end{array}$ & $\begin{array}{l}27,1 \\
(93)\end{array}$ & $\begin{array}{c}0,8 \\
(03)\end{array}$ & - & $\begin{array}{l}2,9 \\
(10)\end{array}$ \\
\hline Cruz das Almas & $\begin{array}{l}59,0 \\
(88)\end{array}$ & $\begin{array}{l}39,6 \\
(59)\end{array}$ & $\begin{array}{c}1,3 \\
(02)\end{array}$ & - & - & $\begin{array}{c}79,8 \\
(107)\end{array}$ & $\begin{array}{l}14,1 \\
(19)\end{array}$ & $\begin{array}{c}0,7 \\
(01)\end{array}$ & $\begin{array}{c}0,7 \\
(01)\end{array}$ & $\begin{array}{c}4,4 \\
(06)\end{array}$ & $\begin{array}{c}65,2 \\
(175)\end{array}$ & $\begin{array}{l}30,2 \\
(81)\end{array}$ & - & - & $\begin{array}{c}4,4 \\
(12)\end{array}$ \\
\hline
\end{tabular}

\footnotetext{
${ }^{1}$ Antes da liberação do parasitoide $\boldsymbol{D}$. longicaudata.

${ }^{2}$ Onde: Da: Doryctobracon areolatus; Aa: Asobara anastrephae; Ua: Utetes anastrephae; Osp: Opius sp.; Dl: Diachasmimorpha longicaudata; (-) parasitismo não observado.
} 
exemplo, tamanho do ovipositor, esse fato também pode ser explicado pela possível preferência dessa espécie nativa por larvas de Anastrepha spp., cuja origem é sul-americana. No entanto, o parasitoide exótico D. longicaudata apresenta uma melhor eficiência de parasitismo e provável preferência por larvas de tefritídeos exóticos, como a mosca-domediterrâneo $\boldsymbol{C}$. capitata.

Dos exemplares de moscas-das-frutas identificados, todos pertenciam à espécie $\boldsymbol{A}$. obliqua. Entre as espécies que efetuaram o parasitismo, $\boldsymbol{D}$. areolatus apresentou-se superior às demais espécies, constatando-se um aumento no índice de parasitismo total de 15,86 para 20,42 e 45,19\% nas respectivas etapas de estudo (Figura 1). As demais espécies encontradas em menor número parasitando larvas de $A$. obliqua em frutos de 'umbu-cajá', U. anastrephae e Opius sp., podem ter sido beneficiadas por hospedeiros, cujas larvas não foram parasitadas pelas espécies predominantes neste trabalho.

Contudo, mais estudos devem ser realizados acerca do estabelecimento desse parasitoide exótico e sua eficiência no controle de moscas-das-frutas em diferentes hospedeiros, nas condições neotropicais, tendo em vista as mudanças climáticas globais e consequentes alterações no agroecossitema.

\section{CONCLUSÃO}

A liberação da espécie exótica $\boldsymbol{D}$. longicaudata não apresenta efeitos negativos na ocorrência dos parasitoides nativos e contribui para complementar o controle biológico natural de $\boldsymbol{A}$. obliqua em frutos de umbu-cajá, nas condições deste estudo.

\section{AGRADECIMENTOS}

Os autores agradecem a colaboração de Dr. Roberto Antonio Zucchi (USP/ESALQ) e Dra. Angélica Penteado Dias (UFSCAR), pela confirmação de alguns exemplares de Braconidae (Alysiinae). À Fundação de Amparo à Pesquisa do Estado da Bahia (FAPESB), pelo apoio financeiro ao projeto e pela concessão de bolsa de Mestrado para Zuzinaide Vidal Bomfim; ao Conselho Nacional de Desenvolvimento Científico e Tecnológico (CNPq), pela Bolsa de Produtividade concedida a Carlos Alfredo Lopes de Carvalho.

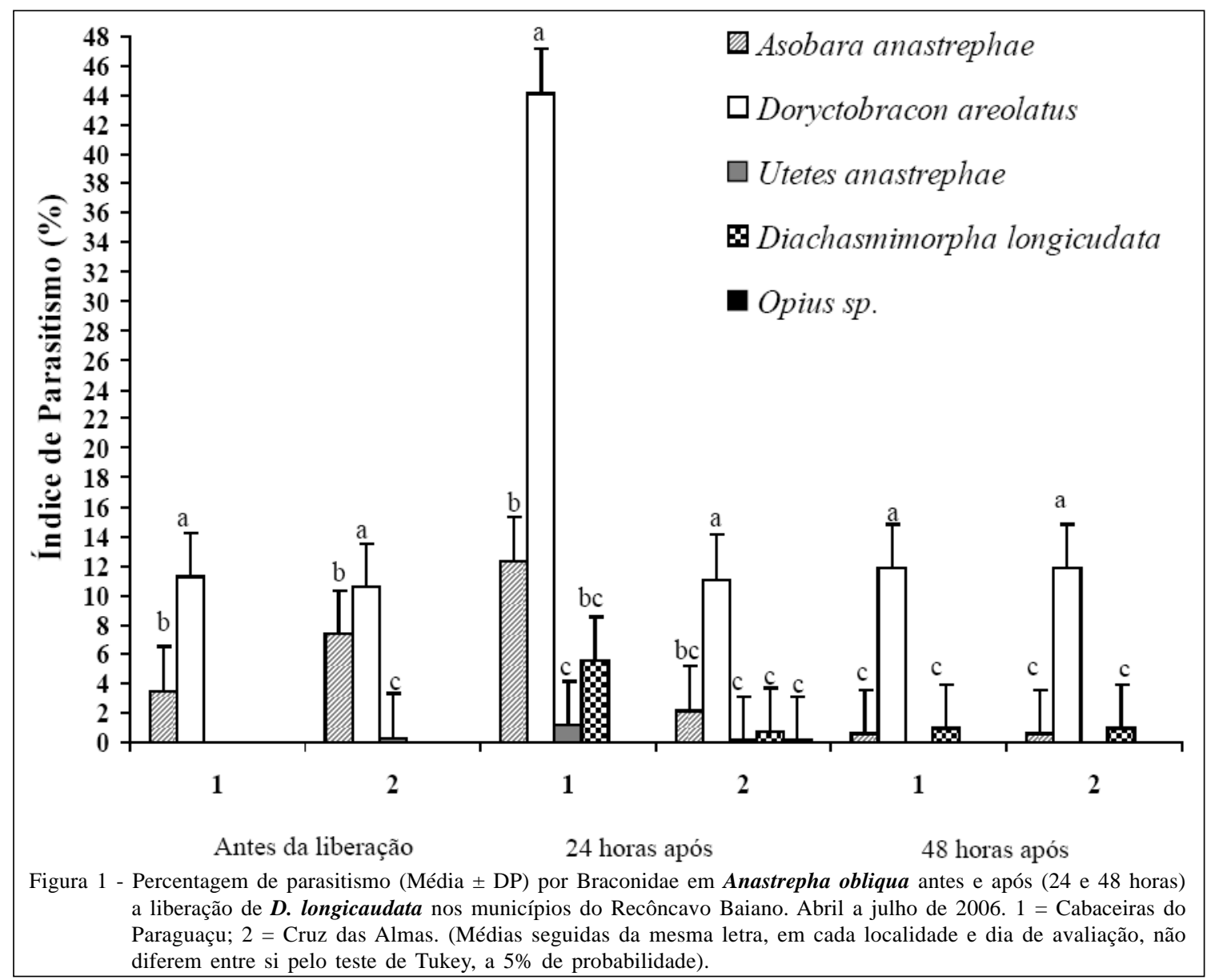




\section{REFERÊNCIAS}

AGRITEMPO. Sistema Nacional de Agrometeorologia. Embrapa Informática Agropecuária - Centro de Pesquisas Metereológicas e Climáticas aplicadas a Agricultura. Disponível em: <http://www.agritempo.gov.br/agroclima/ sumario?uf=BA>. On line. Acesso: março de 2009.

ALVARENGA, C.D. et al. Introdução e recuperação do parasitóide exótico Diachasmimorpha longicaudata (Ashmead) (Hymenoptera: Braconidae) em pomares comerciais de goiaba no Norte de Minas Gerais. Neotropical Entomolgy, v.34, p.133-136, 2005. Disponível em: <http://www.scielo.br/scielo.php?script=sci_arttext\&pid=S1519566X2005000100020\&lng=e\&nrm=iso\&tlng=e $>$. Acesso em: 21 jan. 2008. doi: 10.1590/S1519-566X2005000100020.

BARANOWSKI, R.M. et al. Biological control of the Caribbean fruit fly, Anastrepha suspensa (Loew). Florida Entomologist, v.76, p. 245-250, 1993.

BENNET, F.D. Do introduced parasitoids displace natives ones? Florida Entomolgist, v.76, p.54-63, 1993.

CARVALHO, R. da S. Avaliação das liberações inoculativas do parasitóide exótico Diachasmimorpha longicaudata (Ashmead) (Hymenoptera: Braconidae) em Pomar diversificado em Conceição do Almeida, BA. Neotropical Entomology, v.34, p.799-805, 2005. Disponível em: <http://www.scielo.br/ scielo.php? script =sci_arttext\&pid=S1519566X2005000500012>. Acesso em: 22 dez. 2007. doi: 10.1590/S1519-566X2005000500012.

CARVALHO, R. da S.; NASCIMENTO, A.S. Criação e utilização de Diachasmimorpha longicaudata para controle biológico de moscas-das-frutas. In: PARRA, J.R.P. et al. Controle biológico no Brasil: parasitóides e predadores. São Paulo: Manole, 2002. p.65-179.

DUAN, J.J. et al. Evaluation of the impact of the fruit fly parasitoid Diachasmimorpha longicaudata (Hymenoptera: Braconidae) on non target tephritid, Eutreta xanthochaeata (Diptera: Tephritidae). Biological Control, v.9, p 81-88, 1997.
FERREIRA, D.F. SISVAR - versão 4.6. Lavras: DEX/UFLA, 2004. 32p.

MATRANGOLO, W.J.R. et al. Parasitóides de moscas-dasfrutas (Diptera: Tephritidae) associados a fruteiras tropicais. Anais da Sociedade Entomológica do Brasil, v.27, p.593603, 1998.

PURCELL, M.F. et al. Influence of guava ripening on parasitism of the oriental fruit fly, Bactrocera dorsalis (Hendel) (Diptera: Tephritidae), by Diachasmimorpha longicaudata (Ashmead) (Hymenoptera: Braconidae) and other parasitoids. Biological Control, v.4, p.393-403, 1994.

SANTOS, W. da S. et al. Parasitóides (Hymenoptera: Braconidae) de Anastrepha (Diptera: Tephritidae) associados ao umbu-cajá em Cruz das Almas, Recôncavo Baiano. Magistra, v.20, p.155-160, 2008.

SIVINSK, J. et al. Spatial and temporal distribution of parasitoids of mexican Anastrepha species (Diptera: Tephritidae) within the canopies of fruit trees. Annal Entomological Society America, v.90, p.604-618, 1997.

SOUZA FILHO, M.F. Biodiversidade de moscas-das-frutas (Diptera: Tephritidae) e seus parasitóides (Hymenoptera) em plantas hospedeiras no Estado de São Paulo. 1999. 173f. Dissertação (Mestrado em Entomologia) - Curso de Pósgraduação em Entomologia, ESALQ/USP, São Paulo, SP.

WAAG, J.K.; MILLS, N.L. Understanding and measuring the impact of natural enemies on pest populations. In: MARKHAM, R.H. et al. Biological control manual. Principles and practice of biological control. UNDP/FAO. Cotonou, Republic of Benin. International Institute of Tropical Agriculture, Biological Control Center for África, 1992. V.1, p.84-144.

ZUCCHI, R.A. Taxonomia. In: MALAVASI, A.; ZUCCHI, R.A. Moscas-das-frutas de importância econômica no Brasil: conhecimento básico e aplicado. Ribeirão Preto: FAPESP-Holos, 2000. p.13-24. 